\title{
A PROBABILISTIC PROOF OF THE SERIES REPRESENTATION OF THE MACDONALD FUNCTION WITH APPLICATIONS
}

\section{ASLAM CHAUDHRY and MUNIR AHMAD}

Department of Mathematical Sciences

King Fahd University of Petroleum and Minerals

Dhahran, Saudi Arabia

(Received October 2, 1992 and in revised form August 24, 1993)

ABSTRACT. A series representation of the Macdonald function is obtained using the properties of a probability density function and its moment generating function. Some applications of the result are discussed and an open problem is posed.

KEY WORDS AND PHRASES. Non-central moments, moment generating function, inverse Gaussian distribution.

1991 AMS SUBJECT CLASSIFICATION CODES. 33-02 60E05.

\section{INTRODUCTION.}

Kadell [8] used the probabilistic approach to prove Ramanujan's ${ }_{1} \psi_{1}$ sum. Ismail [6] gave the most natural proof of Ramanujan ${ }_{1} \psi_{1}$ sum that extended to multivariate hypergeometric functions. Some algebraic and other techniques are used to provide simple proofs of the established identities, see $[1,4,5,6,8,9]$. In this paper we have used probabilistic approach to derive a series representation $[2$, p. 100] of the Macdonald function. Some applications of the result are discussed and an open problem is posed.

\section{MAIN RESULTS.}

LEMMA 2.1. For $\operatorname{Re}(z)>0$

$$
\frac{\partial^{n} K_{\alpha}(z)}{\partial \alpha^{n}}= \begin{cases}\int_{0}^{\infty} t^{n} \exp (-z \cosh t) \cosh \alpha t d t & \text { if } n \text { is even } \\ \int_{0}^{\infty} t^{n} \exp (-z \cosh t) \sinh \alpha t d t & \text { if } n \text { is odd }\end{cases}
$$

PROOF. It follows from the integral representation [3, p. 358]

$$
K_{\alpha}(z)=\int_{0}^{\infty} \exp (-z \cosh t) \cosh (\alpha t) d t
$$

of the Macdonald function $K_{\alpha}(z), \quad \operatorname{Re} z>0$. 
THEOREM 2.1. Let $0<t<1$ and $\operatorname{Re} z>0$. Then,

$$
K_{\alpha}(2 z \sqrt{1-t})=(1-t)^{\alpha / 2} \sum_{r=0}^{\infty} K_{\alpha+r}(2 z)(z t)^{r} / r !
$$

PROOF. Let us define the function $I(\alpha, \beta)$ by

$$
I(\alpha, \beta)=\int_{0}^{\infty} x^{\alpha-1} \exp \left(-x-\beta x^{-1}\right) d x, \quad-\infty<\alpha<\infty, \beta>0 .
$$

Then, the function defined by

$$
f(x)=(I(\alpha, \beta))^{-1} x^{\alpha-1} \exp \left(-x-\beta x^{-1}\right), \quad x>0, \beta>0
$$

is the probability density function (pdf). It may be noted that the pdf (2.3) has appeared in an earlier work. This is the limiting case $a \rightarrow 0$ of theorem 1.11 in [7].

The $r$-th non-central moment of the random variable $X$ having (2.3) as its pdf is given by

$$
E\left(X^{r}\right)=\int_{0}^{\infty} x^{r} f(x) d x=\frac{I(\alpha+r, \beta)}{I(\alpha, \beta)} .
$$

The moment generating function (mgf) of $f(x)$ is given by

$$
E\left(e^{t x}\right)=\int_{0}^{\infty} e^{t x} f(x) d x=\frac{1}{I(\alpha, \beta)} \int_{0}^{\infty} x^{\alpha-1} \exp \left(-(1-t) x-\beta x^{-1}\right) d x, \quad 0 \leq t<1 .
$$

Substituting $(1-t) x=\tau$ in (2.5) and simplifying we get

$$
E\left(e^{t x}\right)=\frac{I(\alpha, \beta(1-t))}{(1-t)^{\alpha} I(\alpha, \beta)} .
$$

The relation

$$
E\left(e^{t x}\right)=\sum_{r=0}^{\infty} E\left(X^{r}\right) \frac{t^{r}}{r !}
$$

yields,

$$
\frac{I(\alpha, \beta(1-t))}{(1-t)^{\alpha} I(\alpha, \beta)}=\sum_{r=0}^{\infty} \frac{I(\alpha+r, \beta)}{I(\alpha, \beta)} \frac{t^{r}}{r !}
$$

or

$$
I(\alpha, \beta(1-t))=\left[\sum_{r=0}^{\infty} I(\alpha+r, \beta) \frac{t^{r}}{r !}\right](1-t)^{\alpha} .
$$

It is known $[3$, p. 340] that

$$
I(\alpha, \beta)=2 \beta^{\alpha / 2} K_{\alpha}(2 \sqrt{\beta}), \quad-\infty<\alpha<\infty, \operatorname{Re} \beta>0 .
$$

From (2.7) and (2.8) we get

$$
K_{\alpha}(2 \sqrt{\beta(1-t)})=(1-t)^{\alpha / 2} \sum_{r=0}^{\infty} \beta^{r / 2} K_{\alpha+r}(2 \sqrt{\beta}) \frac{t^{r}}{r !} .
$$

The substitution $\beta=z^{2}$ in (2.9) yields the proof of the theorem.

In particular when $\alpha=0$ and $\beta=z^{2}$ in (2.9) we get

$$
K_{0}(2 z \sqrt{(1-t)})=\sum_{n=0}^{\infty}\left\{z^{n} K_{n}(2 z)\right\} \frac{t^{n}}{n !},
$$


which shows that $K_{0}(z \sqrt{1-t})$ is the generating function of $\frac{1}{n !}(z / 2)^{n} K_{n}(z), \quad n=0,1,2,3, \ldots$.

An immediate consequence of the theorem is the following result which provides the closed form solution to the representation of the first derivative with respect to the order of the Macdonald function at the integral values of the order. The problem remains open for the higher derivatives and the other values of the order of the function.

COROLLARY 2.1. For $\operatorname{Re}(z)>0$,

$$
\frac{\partial}{\partial \alpha}\left[K_{\alpha}(2 z)\right]_{\alpha=n}=\frac{1}{2} \frac{n !}{z^{n}} \sum_{j=1}^{n} \frac{(z)^{n-\jmath} K_{n-\jmath}(2 z)}{j(n-j) !} .
$$

PROOF. Differentiating both sides of (2.1) with respect to $\alpha$ we get

$$
\frac{\partial}{\partial \alpha}\left[K_{\alpha}(2 z \sqrt{1-t}]=(1-t)^{\alpha / 2} \sum_{r=0}^{\infty}\left[\frac{\partial}{\partial \alpha}\left\{K_{\alpha+r}(2 z)\right\}+\frac{1}{2} \ln (1-t) K_{\alpha+r}(2 z)\right] \frac{(t z)^{r}}{r !} .\right.
$$

However, it follows from the lemma that

$$
\frac{\partial}{\partial \alpha}\left[K_{\alpha}(z)\right]_{\alpha=0}=0
$$

Therefore, substituting $\alpha=0$ in (2.10) and using the series representation of $\ln (1-t)$ and (2.11) we get

$$
\sum_{r=0}^{\infty} \frac{\partial}{\partial \alpha}\left[K_{\alpha+r}(2 z)\right]_{\alpha=0} \frac{(z t)^{r}}{r !}=\frac{1}{2}\left(\frac{t}{1}+\frac{t^{2}}{2}+\cdots+\frac{t^{n}}{n}+\cdots\right) \sum_{r=0}^{\infty} K_{r}(2 z) \frac{(z t)^{r}}{r !} .
$$

Equating the coefficients of $t^{n}$ in (2.12) yields the desired proof.

COROLLARY 2.2. For Re $z>0$,

$$
\int_{0}^{\infty} t \exp (-2 z \cosh t) \sinh (n t) d t=\frac{1}{2} \frac{n !}{z^{n}} \sum_{\jmath=1}^{n} \frac{z^{n-\jmath} K_{n-\jmath}(2 z)}{j(n-j) !} .
$$

PROOF. This follows from the lemma and Corrollary 2.1.

In particular when $n=2$ we get

$$
\int_{0}^{\infty} t \exp (-2 z \cosh t) \sinh (2 t) d t=\frac{1}{2}\left[K_{0}(2 z)+2 z K_{1}(2 z)\right]
$$

which does not seem to be known in the literature.

We state here an open problem the solution to which will have far-reaching consequences in the generalization of the inverse Gaussian distribution.

STATEMENT OF THE OPEN PROBLEM. Find the relationship of $\frac{\partial^{n} K_{a}}{\partial \alpha^{n}}$ with the other special functions for $n \geq 2$.

ACKNOWLEDGEMENTS. Private correspondence with Professor M. Rahman at Carleton University, Ottawa, Canada is appreciated. The authors are indebted to the the referee for helpful comments and to the King Fahd University of Petroleum and Minerals for the excellent research facilities. 


\section{REFERENCES}

1. BRESSOUD, D.M. An easy proof of the Rogers-Ramanujan identities, J. Numb. Thy., 16(1983a), 235-241.

2. ERDÉLYI, ET AL., Higher Transcendental Functions, vol. II(McGraw-Hill, 1954).

3. GRADSHTEYN, I.S. and RYZHIK, I.M. Tables of Integrals, Series, and Products (Academic Press, 1980).

4. HIRSHHORN, M.D. A simple proof of Jacobi's two square theorem, Amer. Math. Monthly 92(1985), 579-580.

5. HIRSHHORN, M.D. A simple proof of Jacobi's four-square theorem, Proc. Amer. Math. Soc. 101(1987), 436-438.

6. ISMAIL, M.E.H. A simple proof of Ramanujan's ${ }_{1} \psi_{1}$ sum, Proc. Amer. Math. Soc. $\underline{63}(1977)$, 185-186.

7. ISMAIL, M.E.H. and KELKER, H.D. Special functions, Steiltjes transforms and infinite divisibility, SIAM J. Math. Anal. 10(1979), 884-901.

8. KADELL, K.W.J. A probabilistic proof of Ramanujan's ${ }_{1} \psi_{1}$ sum, SIAM J. Math. Anal. $\underline{18}(1987 \mathrm{a}), 1539-1548$.

9. RAHMAN, M. A simple proof of Koornwinder's additional formula for the little $q$-Legendre polynomials (to appear). 


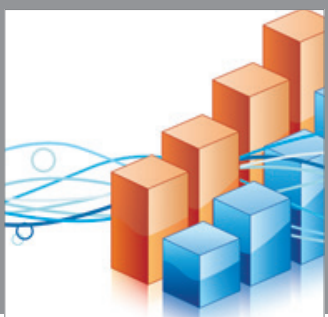

Advances in

Operations Research

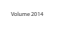

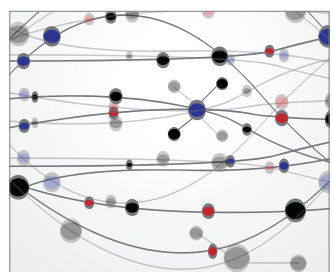

\section{The Scientific} World Journal
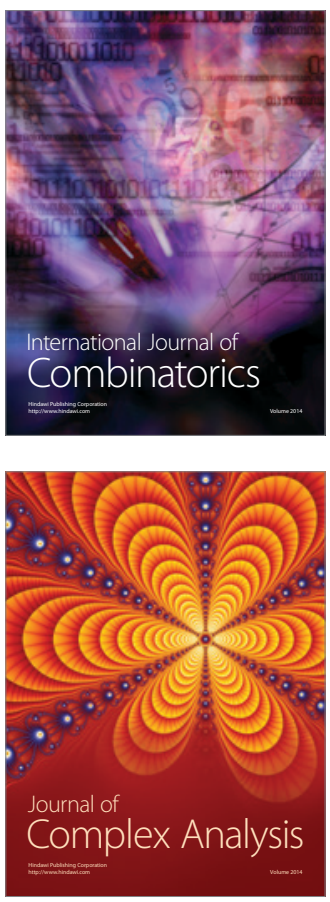

International Journal of

Mathematics and

Mathematical

Sciences
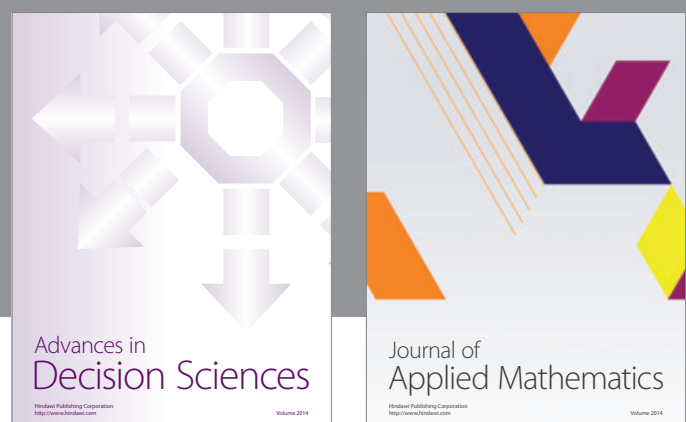

Journal of

Applied Mathematics
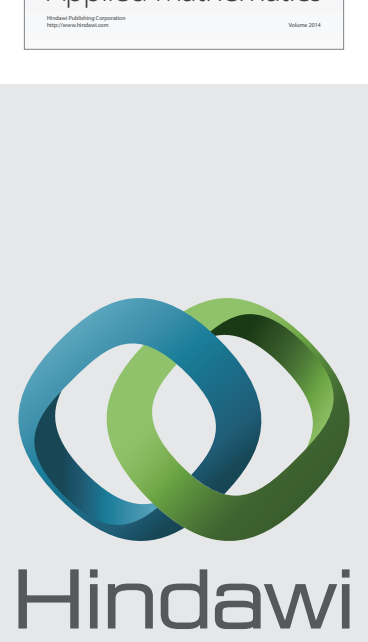

Submit your manuscripts at http://www.hindawi.com
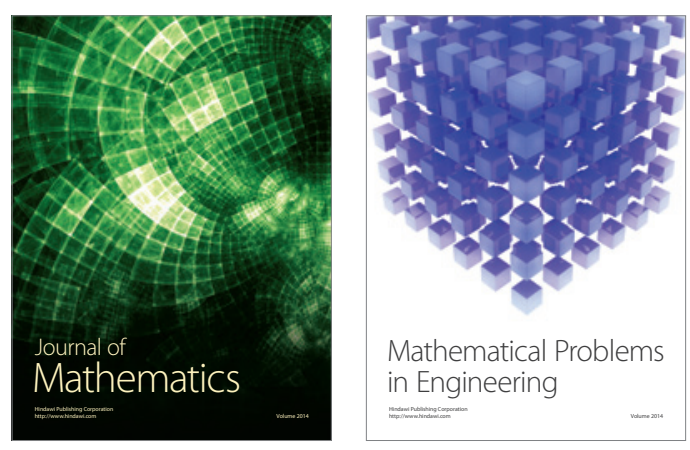

Mathematical Problems in Engineering
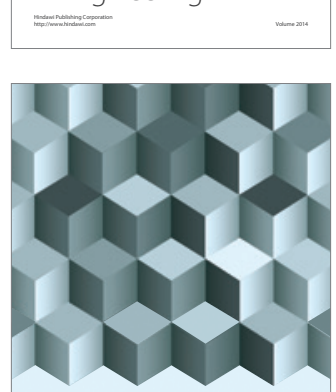

Journal of

Function Spaces
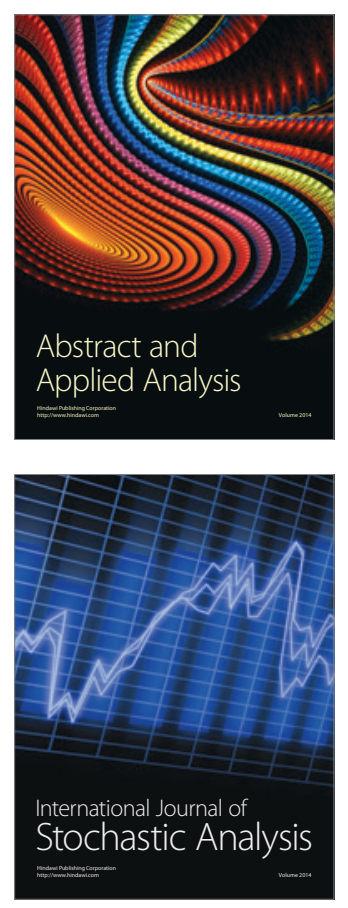

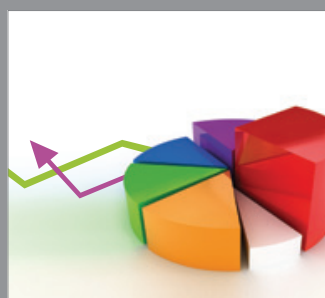

ournal of

Probability and Statistics

Promensencen
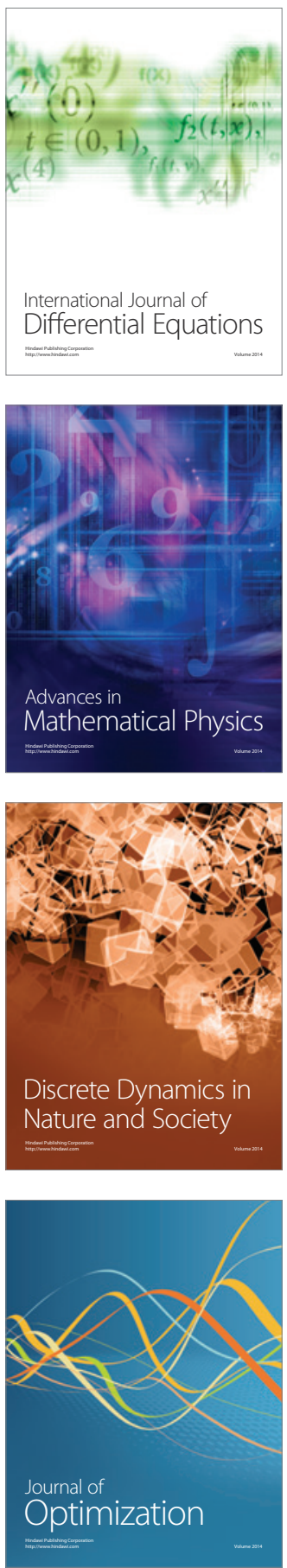